\title{
Breaks and flares in long gamma-ray bursts from massive binaries
}

\author{
Ross P. Church ${ }^{* a}$, Melvyn B. Davies ${ }^{a}$, Andrew J. Levan ${ }^{b}$ and Chunglee Kim ${ }^{c}$ \\ ${ }^{a}$ Lund Observatory, Department of Astronomy and Theoretical Physics, Lund University \\ Box 43, SE-221 00 Lund, Sweden \\ ${ }^{b}$ Department of Physics, University of Warwick \\ Coventry, CV4 7AL, England \\ ${ }^{c}$ Department of Physics, West Virginia University \\ Morgantown, WV 26506, USA \\ E-mail: ross@astro.lu.se, mbdeastro.lu.se, a.j.levan@warwick.ac.uk, \\ chunglee.kimemail.wvu.edu
}

\begin{abstract}
We investigate the observational consequences of a binary model for long-duration gamma-ray bursts, in which the star producing the burst is in a close binary with a massive black-hole companion. This allows tidal interactions within the binary to keep the star spun up, which is necessary in order to form the accretion disc that powers the burst. We model the interaction of the supernova ejecta with the black hole companion and find that there are several robustly predicted features in the resulting accretion on to the disc that powers the gamma-ray burst. First, the accretion history shows a break at around $10^{4} \mathrm{~s}$. This is caused by the presence of the Roche lobe truncating the supply of material to the disc. Secondly, the draining of material that has built up in the disc causes a flare at a few times $10^{4} \mathrm{~s}$. The break time, flare time and total flare fluence show correlations, which owe their origin to a single parameter that determines the timescales present in the system, namely the orbital period of the binary system. Furthermore, we show that the properties of the flares are consistent with those of the late-time flares observed in X-ray light curves of some long-duration gamma-ray bursts.
\end{abstract}

Gamma-Ray Bursts 2012 Conference-GRB2012,

May 07-11, 2012

Munich, Germany

\footnotetext{
*Speaker.
} 


\section{Introduction}

Strong observational evidence links long-duration gamma-ray bursts to type Ic supernovae: the explosions of the stripped cores of massive stars [1]. This lends weight to a scenario where bursts occur following the core collapse of a massive, rapidly-rotating star [2]. The angular momentum of the core leads to a disc forming around the newly-formed black hole, the accretion of which powers a highly relativistic jet that can punch through the relatively rarefied medium at the poles. This scenario suffers from the problem, however, that the strong winds that remove the hydrogen and helium envelopes of the star are expected to also carry away the majority of its angular momentum, spinning the star down below the point at which a disc is expected to form. This problem is particularly hard to avoid for bursts that originate in relatively high-metallicity hosts [3].

One means of overcoming this problem is to spin the exploding star up in a binary $[4,5,6]$. Here the binary's orbit is used as a reservoir of angular momentum. This mechanism has the added advantage that common envelope evolution within the binary provides a natural mechanism to strip off the envelope of the exploding star. It is necessary, however, for this binary to be rather close in the final stages of its evolution, and the companion to be massive [6]. This necessitates a black hole companion of mass $8-10 M_{\odot}$, in an orbit of a few solar radii in extent. In this paper we explore the observable consequences of a gamma-ray burst taking place in such a binary.

\section{Methods}

We first synthesised a representative population of binaries that could be expected to lead to spin-up gamma-ray bursts. We used the population of massive binaries taken from a recent study and extracted those that met the criteria for binary spin up [7]. This supplied us with a set of properties at burst time, in particular stellar masses and semi-major axes. To model the supernova, we utilise simulations of black hole formation during supernovae from the literature [8]. This study finds that some of the material ejected from the star in the early stages of the explosion is stalled by a reverse shock. It subsequently falls back onto the newly-formed compact object, contributing roughly half its mass. The authors obtain an accretion rate on to the central object which we fit as

$$
\dot{M}= \begin{cases}\kappa & 100 \mathrm{~s}<t<t_{\text {plateau }} \\ \kappa\left(t / t_{\text {plateau }}\right)^{-5 / 3} & t>t_{\text {plateau }},\end{cases}
$$

with $t_{\text {plateau }} \simeq 260 \mathrm{~s}$. To investigate the effect of the companion on this material as it is ejected and falls back we utilise a reduced three-body simulation. We place a large number of test particles in a sphere surrounding the exploding star, and eject them with a velocity distribution chosen so that, in the absence of a companion, they would fall back at the rate given by Equation 2.1.

Our binary is symmetric under reflection in the orbital plane, so we would expect an equal flux of mass and momentum in falling back material in both directions perpendicular to the plane at any given time. As a result, this material will collide in the plane and form a disc. We expect material falling into such a disc to collide and circularise within the orbital timescale of the disc. If in the process of circularising it collides with material already present in the disc then we merge the particles representing the two sets of material and circularise the resulting, more massive, particle at the radius implied by its new specific angular momentum. Once material has circularised we 
allow it to accrete inwards via viscous transport of angular momentum outwards through the disc. We model the disc with a simple prescription from the literature [9]. The rate of radial inflow of material at radius $r$ is given by $\dot{r}=-r \alpha \Omega_{\mathrm{Kep}} \tilde{h}^{2}$, where $\Omega_{\mathrm{Kep}}$ is the orbital angular frequency at that point in the disc, $\alpha=0.1$, and we assume that the ratio of disc scale height to radius, $\tilde{h} \equiv H_{\text {disc }} / R_{\text {disc }}=0.1$. When material arrives at the last stable orbit around the black hole it is accreted.

There is still considerable uncertainty over whether black holes receive velocity kicks at birth analogous to those of neutron stars. In some cases, for example the black hole in Cygnus X-1, there is strong evidence that they do not, whereas the Galactic orbits of some other X-ray binaries imply that they have received kicks similar to those of neutron stars $[10,11]$. For the black holes that we are considering it is likely that a neutron star forms initially, and that subsequent fallback transforms it into a black hole. Thus we would expect a kick of equal momentum to a typical neutron star kick. For a $4.5 M_{\odot}$ black hole this translates to a kick velocity of roughly $100 \mathrm{~km} / \mathrm{s}$ [12]. Therefore, in addition to unkicked models we consider a set of models where the newlyformed black hole is kicked by $100 \mathrm{~km} / \mathrm{s}$ in the $\pm x, y$ directions. These models give results that are not clearly different from the unkicked results.

\section{Results}

\subsection{A typical system}

Figure 1 illustrates a binary that exemplifies the behaviour of the majority of our models. The left-hand panel shows the positions at which material reaches the plane, and hence accretion discs, around the two stars. At early times the material falling back lands very close to the star, and the accretion rate (right-hand panel) is very close to the single-star case. As the exploding star (red) moves anti-clockwise round its orbit, the material has been increasingly affected by the force of the companion and hence lands in the disc at larger distances from the newly-formed star. Eventually the fallback positions are far enough away from the star to be outside its Roche lobe, and no more material is accreted. This corresponds to the break in the accretion curve that can be seen at a time of roughly $8000 \mathrm{~s}$. Later, some material falls back on to the companion star. That, combined with material that has entered the outer part of the accretion disc, forms the flare that is seen centred round a time of $30000 \mathrm{~s}$.

\subsection{Flare properties}

In Figure 2 we plot some of the properties of the flares that occur in our models. The left-hand panel shows the time of the break in the accretion curve, $t_{\text {break }}$, plotted as a function of the time of maximum flare luminosity, $t_{\text {flare}}$. There is a clear positive correlation despite some scatter: later flares come from systems in which the accretion break occurs at a later time. Whilst this is in a sense obvious, simply requiring the flare to take place after the accretion break without any further constraint would lead to a much less pronounced correlation.

The right-hand panel of the figure shows the total energies in the flares. Again a correlation is visible, although less strong in this case: later flares have less energy. The three pink points are the isotropic energies in three long-duration gamma-ray burst flares that occur at late times [13]. These 

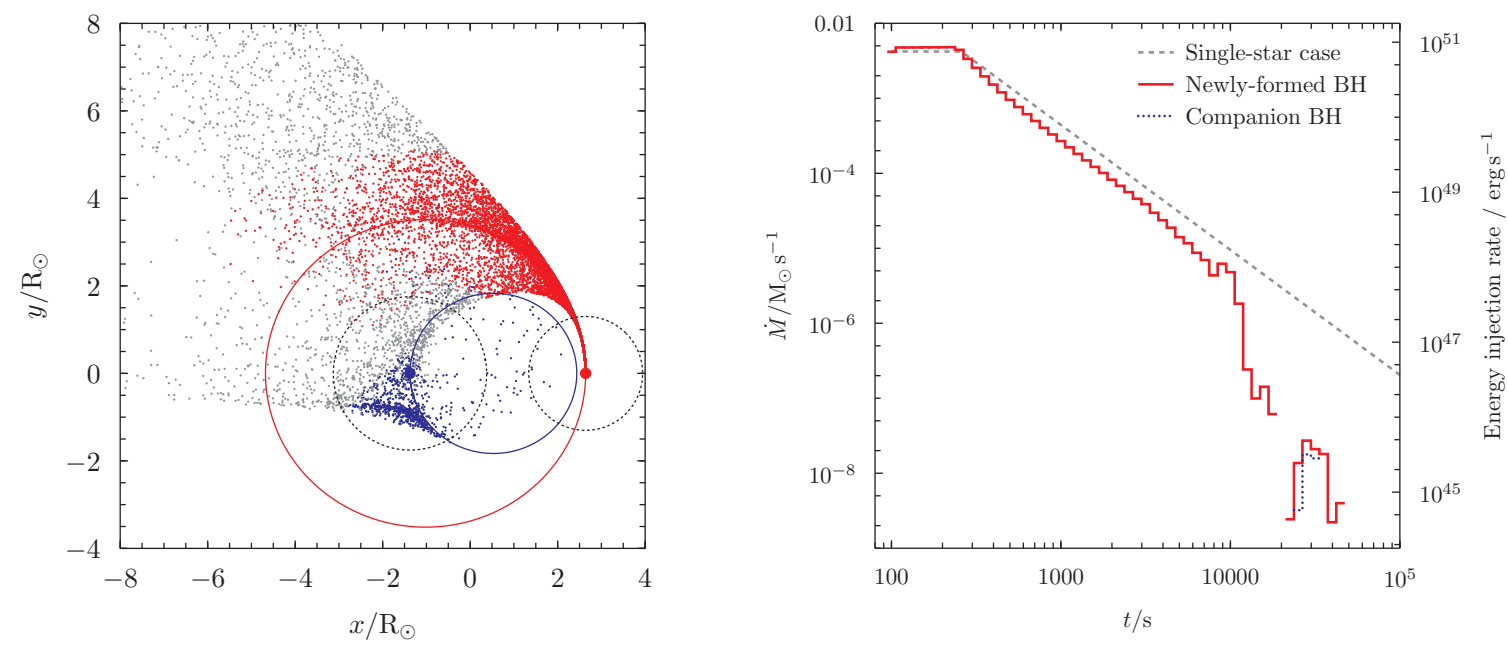

Figure 1: A typical model. Left: Positions at which particles enter the discs. The solid red point (right) is the initial position of the exploding star. Red dots are particles that fall into its Roche lobe. The solid blue point (left) is the black-hole companion. Blue dots fall into its Roche lobe. Grey dots are material that falls back outside the Roche lobes. Red and blue lines show the stellar orbits and dotted circles the initial Roche-lobe radii. Right: The accretion rates on to the two stars as a function of time. The red line shows accretion on to the exploding star, the blue dotted line that for the companion. The grey dashed line is the single-star case. The right-hand ordinate gives the accretion luminosity if ten per cent of the accreted mass is released as energy.
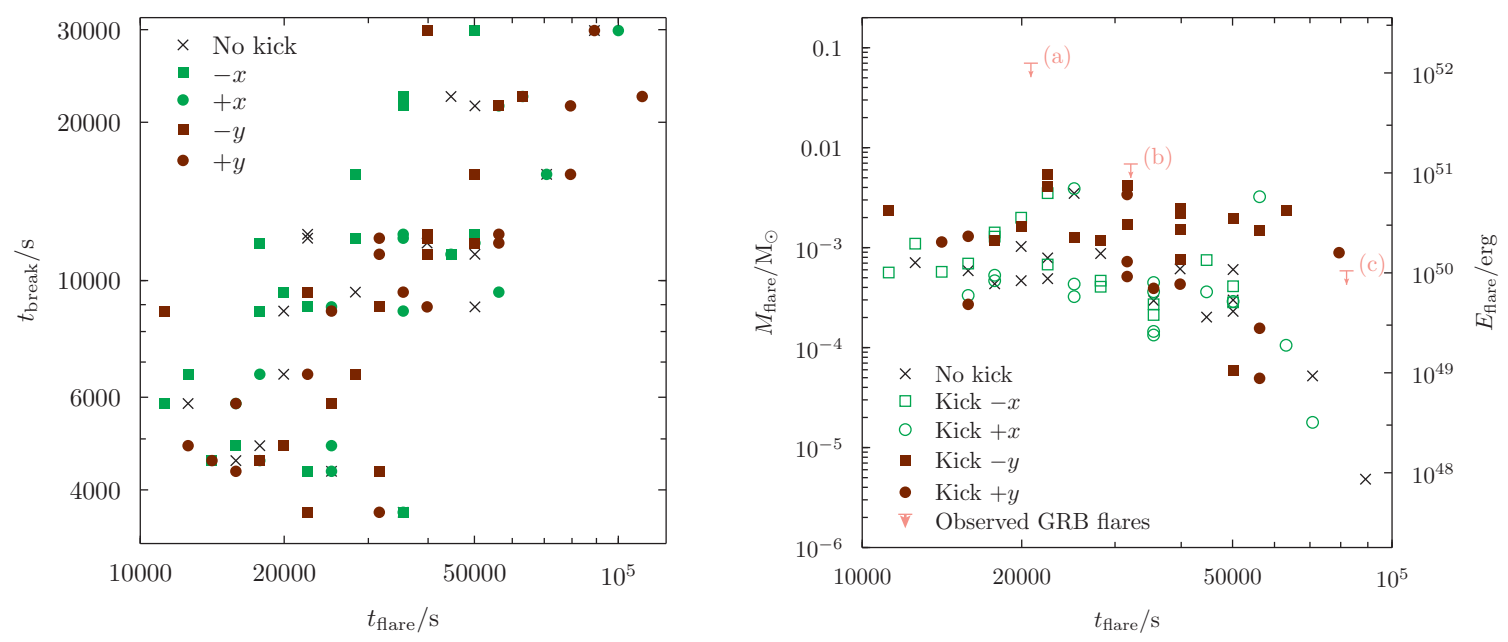

Figure 2: Properties of late-time flares in spin-up binary GRB models. Left: the time of the pre-flare break in the accretion curve, $t_{\text {break }}$, plotted as a function of time of flare, $t_{\text {flare }}$. Black crosses show unkicked models, green open circles and squares kicks in the $+x$ and $-x$ directions, brown filled circles and squares kicks in the $+y$ and $-y$ directions. Right: mass of material in the flare, $M_{\text {flare }}$, plotted as a function of $t_{\text {flare }}$. Colours as in LH panel. The right-hand ordinate shows the total energy in the flare assuming a $10 \%$ conversion from mass to energy. The pink symbols show the isotropic energy in late-time flares from three long gamma-ray bursts: (a) GRB 050502B, (b) GRB 070107 and (c) GRB 070318. 

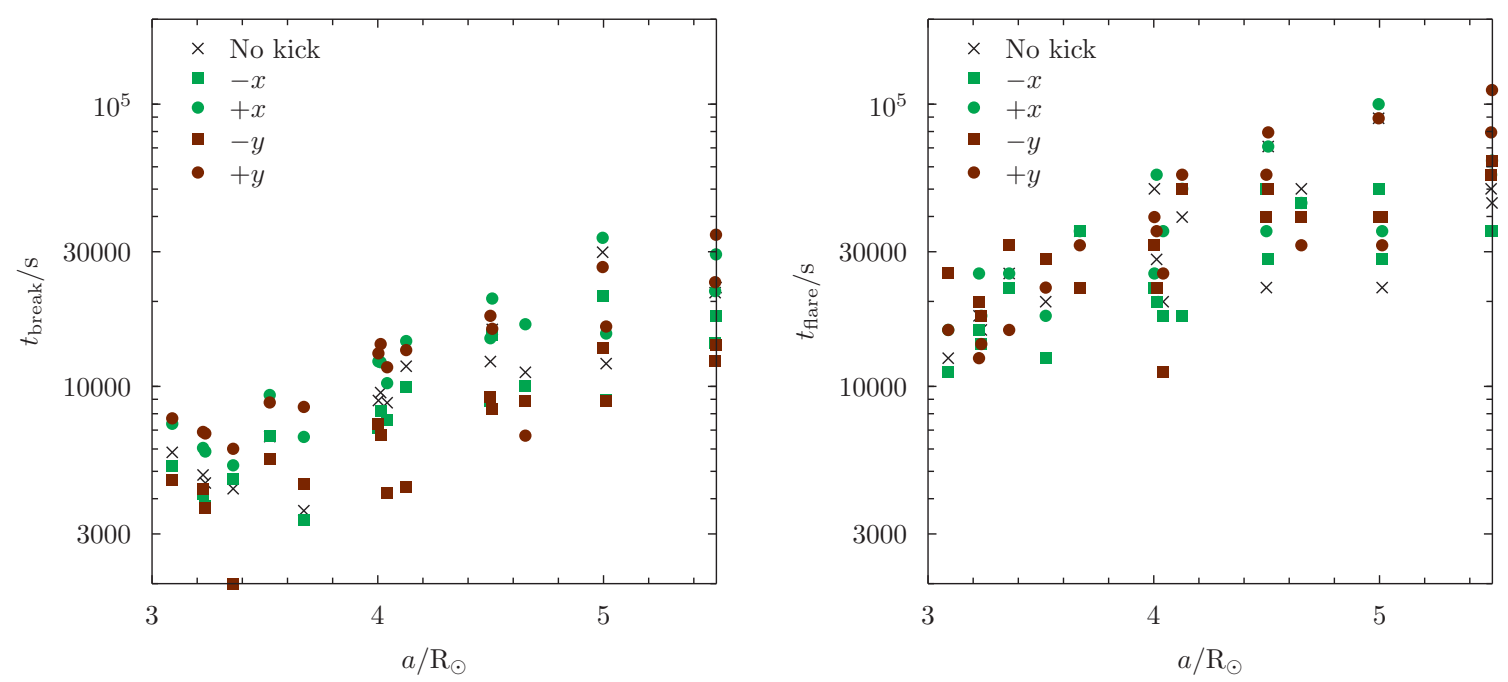

Figure 3: Left: time of accretion break, $t_{\text {break }}$ as a function of binary semi-major axis before supernova, $a$. Right: time of maximum flare luminosity, $t_{\text {flare }}$, as a function of $a$. Symbols and colours as in Figure 2 .

points show a similar trend of declining fluence with flare time. Although they are systematically above our predictions, this assumes that the emission is isotropic. Beaming to roughly $1 / 30$ of the sky would make the observed fluences consistent with our predictions.

\subsection{Physical origin of the observed trends}

There is a single timescale that influences both the break and the flare, namely the orbital period of the binary. This leads to the correlations visible in Figure 2. For wider binaries, with a longer orbital period, the critical radius that material must travel out to before being affected by the companion increases. Hence only material falling back at later times is affected, and the break and flare occur later. This is borne out by Figure 3, which shows the correlations between the semi-major axes of our binaries and the break and flare times. Although there is some scatter, there is a distinct positive correlation between the semi-major axis $a$ and break times (left panel), as well as between $a$ and flare times (right panel). Similarly, for flares occurring at later times there is a smaller fraction of the material left to accrete (see Figure 1). Thus flares at later times, when there is less material left in the binary to accrete, have a smaller fluence.

The origin of break and flare times, and flare mass, in the orbital properties of the binary means that they are determined by the criterion that we started with; namely, that the binary be close enough to be spun up by tidal interactions at a late time in its evolution. This sets a natural time of $10^{4}-10^{5} \mathrm{~s}$ for the processes that we predict. This does mean, however, that we cannot explain early flares in this manner. They must have an origin in some other mechanism, for example, gravitational instability leading to lumps forming in the accretion disc.

\section{Acknowledgements}

RPC is funded by a Marie-Curie Intra-European Fellowship, grant No. 252431, under the European Commission's FP7 framework. CK acknowledges a Marie-Curie International Incom- 
ing Fellowship under the European Commission's FP7 framework. This work was supported by the Swedish Research Council (grants 2008-4089 and 2011-3991). The calculations presented in this paper were carried out using computer hardware purchased with grants from the Royal Fysiographic Society of Lund. This work made use of data supplied by the UK Swift Science Data Centre at the University of Leicester.

\section{References}

[1] J. Hjorth and J. S. Bloom. The Gamma-Ray Burst - Supernova Connection. [astro-ph/1104.2274], 2011.

[2] S. E. Woosley. Gamma-ray bursts from stellar mass accretion disks around black holes. ApJ, 405:273-277, 1993.

[3] E. M. Levesque, L. J. Kewley, J. F. Graham, and A. S. Fruchter. A High-metallicity Host Environment for the Long-duration GRB 020819. ApJ, 712:L26-L30, 2010.

[4] P. Podsiadlowski, P. A. Mazzali, K. Nomoto, D. Lazzati, and E. Cappellaro. The Rates of Hypernovae and Gamma-Ray Bursts: Implications for Their Progenitors. ApJ, 607:L17-L20, 2004.

[5] R. G. Izzard, E. Ramirez-Ruiz, and C. A. Tout. Formation rates of core-collapse supernovae and gamma-ray bursts. MNRAS, 348:1215-1228, 2004.

[6] A. J. Levan, M. B. Davies, and A. R. King. Neutron star binaries and long-duration gamma-ray bursts. MNRAS, 372:1351-1356, 2006.

[7] R. P. Church, A. J. Levan, M. B. Davies, and N. Tanvir. Implications for the origin of short gamma-ray bursts from their observed positions around their host galaxies. MNRAS, 413:2004-2014, 2011.

[8] A. I. MacFadyen, S. E. Woosley, and A. Heger. Supernovae, Jets, and Collapsars. ApJ, 550:410-425, 2001.

[9] R. Perna, P. J. Armitage, and B. Zhang. Flares in Long and Short Gamma-Ray Bursts: A Common Origin in a Hyperaccreting Accretion Disk. ApJ, 636:L29-L32, 2006.

[10] I. F. Mirabel and I. Rodrigues. Formation of a Black Hole in the Dark. Science, 300:1119-1121, 2003.

[11] S. Repetto, M. B. Davies, and S. Sigurdsson. Investigating stellar-mass black hole kicks. [astro-ph/1203.3077], 2012.

[12] B. M. S. Hansen and E. S. Phinney. The pulsar kick velocity distribution. MNRAS, 291:569-577, 1997.

[13] P. A. Curran, R. L. C. Starling, P. T. O'Brien, O. Godet, A. J. van der Horst, and R. A. M. J. Wijers. On the nature of late X-ray flares in Swift gamma-ray bursts. A\&A, 487:533-538, 2008. 